\title{
Modification of the Pointe Scientific Automated Glucose-6-Phosphate Dehydrogenase (G6PD) Assay for High-Throughput Use in the Clinical Laboratory
}

\author{
Erin Elizabeth Milner*, Absone Rowland, Jessica Jeanette Fasio, Lam Henry \\ Department of Pathology, Dwight D. Eisenhower Army Medical Center, Ft Gordon, United States of America
}

Email address:

erin.e.milner.mil@mail.mil (E.E. Milner), absone.p.rowland.civ@mail.mil (A. Rowland), jessica.j.fasio.mil@mail.mil (J. J. Fasio), henry.lam.civ@mail.mil (L. Henry)

${ }^{*}$ Corresponding author

\section{To cite this article:}

Erin Elizabeth Milner, Absone Rowland, Jessica Jeanette Fasio, Lam Henry. Modification of the Pointe Scientific Automated Glucose-6Phosphate Dehydrogenase (G6PD) Assay for High-Throughput Use in the Clinical Laboratory. Clinical Medicine Research.

Vol. 7, No. 5, 2018, pp. 119-123. doi: 10.11648/j.cmr.20180705.13

Received: August 27, 2018; Accepted: September 13, 2018; Published: November 12, 2018

\begin{abstract}
Herein we report a modified Pointe Scientific method for Glucose-6-Phosphate Dehydrogenase (G6PD) testing which eliminates time-consuming and labor intensive pre-analytical steps previously required. Pre-lyse and on-board lyse Pointe Scientific methods were compared to the on-board lyse Trinity Biotech method using Abbott Architect c8000 analyzers integrated into an a3600 track system. In order to streamline the implementation of this method involving both G6PD and hemoglobin analysis, we sought to capitalize on the Instrument Manager (IM) middleware and the a3600 Abbott track system for high-throughput analysis using the Abbott Architect c8000 platform. IM rules were developed to route the whole blood specimen to the wait area of the Input Output Module (IOM) while the initial result is determined. When the initial result is intermediate or deficient, the G6PD activity per gram of hemoglobin $(\mathrm{U} / \mathrm{g} \mathrm{Hgb})$ is determined by rerouting the specimen to the analyzer as an automated reflex test. The data associated with method comparison, precision, carryover and linearity studies completed per regulatory guidelines indicate the assay parameters reported herein are available for the immediate implementation of fully-automated G6PD testing. A viable method that includes on-board lysing and reflex testing has been implemented allowing for high-throughput G6PD testing in a clinical laboratory. Given market research yielded limited vendors with Food and Drug Administration (FDA)-cleared G6PD assays, the automation of the Pointe Scientific method will allow Department of Defense (DOD) laboratories to rapidly screen service members in order to ascertain the tolerability of a Primaquine dosing regimen.
\end{abstract}

Keywords: Glucose-6-Phosphate Dehydrogenase (G6PD) Assay, Primaquine G6PD Deficiency, Pointe Scientific Automated G6PD Assay

\section{Introduction}

Primaquine $(\mathrm{PQ})$ remains a crucial drug for radical cure and presumptive anti-relapse therapy (PART or terminal prophylaxis) of $\mathrm{P}$. vivax and $\mathrm{P}$. ovale infections while aiding in primary prevention. $[1,2]$ PQ kills the hepatic stages of all human malaria parasites and is the only FDA-approved drug active against the dormant hypnozoite stages of $\mathrm{P}$. vivax and P. ovale. [3] PQ can be used as a control measure to render P. falciparum gametocytes non-infective to mosquitoes and therefore inhibit transmission. [4]
Patients should be tested for glucose-6-phosphate dehydrogenase (G6PD) deficiency before being prescribed PQ for radical cure, terminal prophylaxis, or chemoprophylaxis due to known liabilities associated with hemolytic reactions in patients with deficiencies [2, 5-7]. This potential liability has led to mandatory G6PD testing for service members to ensure deployment readiness. A survey of commercially available G6PD assays for high-throughput screening in the clinical laboratory yields two viable vendors, 
Pointe Scientific and Trinity Biotech.

While DDEAMC initially implemented the Trinity Biotech method, we were informed that manufacturing would discontinue beyond 2018. Pointe Scientific manufactured a G6PD reagent set, hemoglobin reagents and hemoglobin standard Food and Drug Administration (FDA)-cleared for use in the clinical laboratory, but unfortunately the published method involved pre-lysing the whole blood specimen prior to automated analysis. Given the projected workload, we sought to fully automate the Pointe Scientific method. To this end we report a viable on-board lysing method that allows for highthroughput analysis of whole blood loaded onto the a3600 Abbott track system with complementary utilization of the wait area and reflex testing to quantify G6PD activity per $\mathrm{U} / \mathrm{g} \mathrm{Hb}$.

\section{Materials and Methods}

\subsection{Instrumentation}

All specimens were analyzed utilizing an Abbott Architect c8000 analyzer attached to an a3600 Abbott track system.

\subsection{Pointe Scientific Pre-Lyse G6PD Assay Procedure}

Reagents (product code G7583-180) and pre-lysed specimens were prepared according to the package insert. [8]

\subsection{Pointe Scientific On-Board-Lyse G6PD Assay Procedure}

(1) Reagent Preparation: Prepare according to the pre-lyse procedure. [8]

(2) Lyse Preparation: The Pointe Scientific lyse was transferred into a separate $90 \mathrm{~mL}$ reagent cartridge and loaded onto the Architect analyzer. Both G6PD reagent mixture $(\mathrm{R} 1+\mathrm{R} 2)$ and the G6PD lyse were manually assigned a carousel location in the reagent status screen Note both Pointe Scientific and Abbott software utilize the nomenclature R1 and R2. The Pointe Scientific R1 and R2 refers to the kit reagent bottles used to formulate the reagent mixture. The Abbott Architect R1 and R2 refer to the carousel locations (reagent mixture and lyse).

(3) Sample Preparation: Whole blood was collected with ethylenediaminetetraacetic acid (EDTA) specimen collection tubes. Specimen collection tubes were placed on a rocker for 5 minutes before loading the specimens into the a3600 Input Output Module (IOM). Clotted and/or hemolyzed specimens were rejected.

(4) Pointe Scientific Hemoglobin Reagent (H75064-500) [9] and Standard (506-HGB STD) were prepared according to the package inserts. [10] Calibration is required a minimum of bi-annually, but was performed with each change of reagent lot number and completion of major maintenance tasks. The calibration was further verified with each level of control material. If the control results exceeded acceptable ranges, recalibration was completed.

(5) Trinity Biotech G6PD Assay: reagents (product code
345-5) and specimens were prepared according to the package insert. [11]

(6) Quality Control: G6PD was monitored daily using either Trinity Biotech (product codes G 5888, G 5029 and G 6888) or Analytical Control Systems (product codes HC-108, HC-108IN and HC-108DE) normal, intermediate, and deficient quantitative G6PD activity ranges using lot-specific information and preparations according to the package inserts. [12] Verification of the cut-off qualitative G6PD values (normal, intermediate and deficient) were accomplished daily using the QC material. Hemoglobin (HGB) was monitored daily using two levels of BIORAD bilevel diabetes controls (BR740) using the preparation and QC ranges indicated in the package insert. [13]

(7) Analytical Measurement Range (AMR): The AMR was established using three commercially-available controls with subsequent dilutions to generate five data points.

(8) Carryover: Carryover studies were completed using five specimens per the Abbott procedure. [14] It should be noted the instrument creates 'blank' cuvettes to continuously monitor carryover as well.

(9) Assay Parameters: The Abbott Architect assay parameters outlined in the manufacturer's package insert were utilized without modification for the Trinity Biotech [15] and the pre-lyse Pointe Scientific [16] methods. Table 1 provides the Abbot Architect assay parameters utilized for the modified on-board-lyse Pointe Scientific method, which can be programmed in the $\mathrm{c} 8000$ for immediate implementation.

(10)Calculations and data analysis: G6PD activity expressed as $\mathrm{U} / \mathrm{g}$ hemoglobin $(\mathrm{Hb})$ was calculated utilizing the laboratory information system: G6PD $(\mathrm{U} / \mathrm{g} \mathrm{Hb})=\mathrm{G} 6 \mathrm{PD}$ activity $(\mathrm{U} / \mathrm{L}) /$ (hemoglobin concentration $\mathrm{g} / \mathrm{dl} \times 10 \mathrm{dl} / \mathrm{L}$ ). [8] The percent carry over (\% CO) was calculated using the following equation: $\% \mathrm{CO}=[($ pos $5-($ average of pos $1+$ pos $2+$ pos 3)/pos 4] x 100. [14]

Data analysis and graphical representations were generated using EP Evaluator or Microsoft Excel.

\section{Results}

The modifications outlined allowed for the fully automated G6PD analysis of whole blood specimens using an Abbott c8000 analyzer. As shown in Table 2, comparison of both the manual and automated Pointe Scientific methods to the automated Trinity Biotech method yielded correlation coefficients of 0.9666 and 0.9978 , respectfully. As shown in Table 3 precision studies utilizing deficient, intermediate and normal G6PD modalities yielded a percent coefficient of variation $(\% \mathrm{CV})$ of $0.8,0.8$ and $0.7 \%$, respectfully, for patient specimens and $1.5,3.1$, and $0.8 \%$, respectfully, for control materials. The pre-lyse method corresponded to less favorable 9.2, 2.8, and $2.5 \% \mathrm{CV}$ for deficient, intermediate and normal G6PD modalities, respectfully, for within day precision. [8] 
Table 1. Abbott Architect c8000 method parameters for Pointe Scientific pre-lyse (package insert) and on-board-lyse Pointe Scientific (modified) Assays.

\begin{tabular}{|c|c|c|}
\hline & Pointe Scientific Package Insert & Pointe Scientific Modified Parameters \\
\hline \multicolumn{3}{|c|}{ GENERAL- REACTION DEFINITION } \\
\hline Type & Photometric & Photometric \\
\hline \multicolumn{3}{|l|}{ Reaction Definition } \\
\hline Reaction Mode & Rate up & Rate up \\
\hline Primary & 340 & 340 \\
\hline Secondary & None & 700 \\
\hline Read Time Main & $16-33$ & $10-27$ \\
\hline Read Time Flex & $10-17$ & --- \\
\hline Last Read & 33 & 33 \\
\hline Absorbance Range & $0.0000-3.2000$ & $0.0000-3.2000$ \\
\hline Color Correction & None & None \\
\hline Sample Blank Type & None & None \\
\hline \multicolumn{3}{|c|}{ GENERAL - REAGENT/SAMPLE } \\
\hline Reagent & G6PD & G6PD \\
\hline Reagent Volume (R1)* & $250 \mathrm{ul}$ & 160ul \\
\hline \multicolumn{3}{|l|}{ Reagent Volume (R2)* } \\
\hline Diluent & Saline & Point Scientific G6PD Lyse \\
\hline Sample & 9.0 & 10.0 \\
\hline Diluted Sample & --- & 5.6 \\
\hline Diluent & --- & 90 \\
\hline Dilution Factor & $1: 1$ & $1: 10$ \\
\hline Rate Linearity & 10 & 10 \\
\hline \multicolumn{3}{|l|}{ CALIBRATION } \\
\hline Calibration Factor & 46260 & 4036.0 \\
\hline Blank & water & water \\
\hline Replicates & 3 & 3 \\
\hline Blank Water Sample Dilution & 9.0 & 5.6 \\
\hline Calibration Intervals & 720 hours & 168 hours \\
\hline Blank Absorbance Range & ---- & $0.000-0.0020$ \\
\hline \multicolumn{3}{|l|}{ SMARTWASH } \\
\hline Sample Probe & Water & Water \\
\hline Cuvette Trig & $10 \%$ Detergent B & $10 \%$ Detergent B \\
\hline Cuvette Trig FLUID & $10 \%$ Detergent B & $10 \%$ Detergent B \\
\hline
\end{tabular}

Table 2. Method Comparison Regression Analysis of on-board lyse Trinity Biotech to pre-lyse and on-board-lyse Pointe Scientific Assays.

\begin{tabular}{llllll}
\hline Trinity Biotech Automated Method & Pointe Scientific Methods & N & Slope & Intercept & Correlation Coefficient (R) \\
\hline G6PD automated on-board lyse & G6PD automated on-board lyse & $20 / 20$ & 0.910 & -4.87 & 0.9978 \\
G6PD automated on-board lyse & G6PD manual pre-lyse & $20 / 20$ & 0.739 & 2.3 & 0.9666 \\
\hline
\end{tabular}

Table 3. Automated On-board-lyse Pointe Scientific Precision Studies Utilizing Control and Patient Specimens.

\begin{tabular}{lllll}
\hline Sample & Number of Specimens & Mean* & Standard Deviation (SD)* & Coefficient of Variation (CV) \\
\hline G6PD Deficient (patients) & 20 of 20 & $446.1(444.4$ to 447.9$)$ & 3.8 (2.9 to 5.5$)$ & $0.8 \%$ \\
G6PD Deficient (controls) & 20 of 20 & $128.3(127.4$ to 129.2$)$ & $1.9(1.5$ to 2.8$)$ & $1.5 \%$ \\
G6PD Intermediate (patients) & 20 of 20 & $657.4(655.0$ to 659.8$)$ & $5.1(3.9$ to 7.5$)$ & $0.8 \%$ \\
G6PD Intermediate (controls) & 20 of 20 & $676.3(666.4$ to 686.2$)$ & $21.1(16.0$ to 30.8$)$ & $3.1 \%$ \\
G6PD Normal (patients) & 20 of 20 & $1900.0(1893.8$ to 1906.1$)$ & $13.2(10.0$ to 19.2$)$ & $0.7 \%$ \\
G6PD Normal (controls) & 20 of 20 & $2088.8(2080.6$ to 2096.9$)$ & $17.3(13.2$ to 25.3$)$ & $0.8 \%$ \\
\hline
\end{tabular}

*95\% Confidence Intervals are shown in parentheses

As shown in Figure 1, the linearity of G6PD was analyzed within a measured range of 32.500 to $1400.500 \mathrm{mg} / \mathrm{dL}$ with an $\mathrm{R}^{2}$ of 0.9985 . A carry-over study was completed to investigate whether the whole blood was contaminating the subsequent specimen. The percent carryover was calculated as $-0.33 \%$, which is within the manufacturer's recommended threshold of less than $1.5 \%$. [14]. 


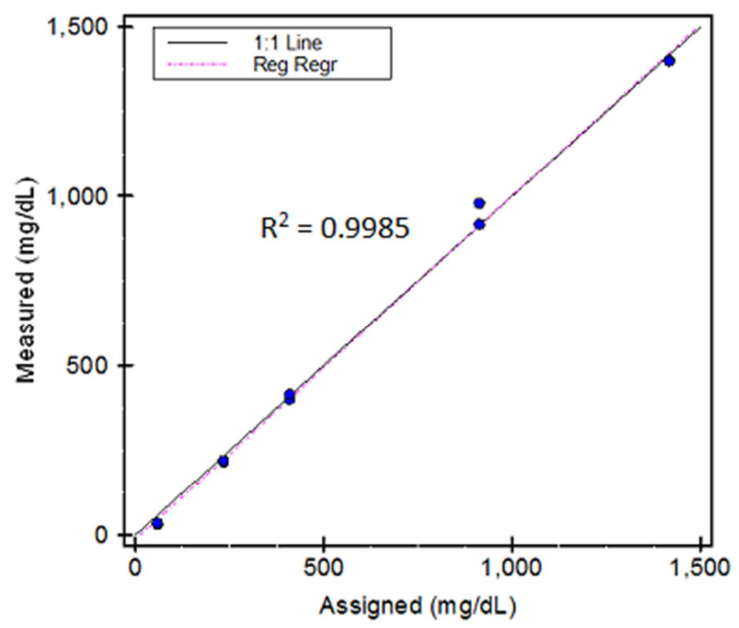

\section{Linearity Summary}

\begin{tabular}{llll}
\hline & N & Slope & Intercept Error \\
\hline Reg. Regression & 5 & $1.014 \pm 0.023$ & $-13.770 \pm 18.04325 .545$
\end{tabular}

Figure 1. Automated On-board Lyse Pointe Scientific Linearity Studies.

\section{Conclusion}

The modified Pointe Scientific assay parameters can be immediately implemented in the clinical laboratory setting using the Abbott Architect c8000 platform, which eliminates the previous requirement for manual lysing and wait time. The subsequent method comparison studies demonstrate the on-board-lyse assay modifications are commensurate with the pre-lyse assay parameters and compare within statistical parameters to the pre-lyse Trinity Biotech method.

The workload was further streamlined by utilizing Instrument Manager (IM) and the a3600 Abbott track system. IM rules were developed to route the whole blood specimen to the wait area of the Input Output Module (IOM) while the initial result is determined. When the initial result is intermediate or deficient, the G6PD activity per gram of hemoglobin $(\mathrm{U} / \mathrm{g} \mathrm{Hgb})$ is determined by rerouting the specimen to the analyzer as an automated reflex test.

To reduce the potential for whole blood contamination, two additional wash methods were implemented. A small wash cycle was programmed to occur during the scheduled pause downtime and a weekly 25 minute wash cycle with Detergent B was implemented during evening maintenance.

The College of American Pathologists (CAP) requirements for modifying FDA-cleared test methods were considered along with quality assurance requirements [17-18]. The following CAP checklist items are of particular relevance and should be considered in the context of all applicable checklist requirements: COM 10000, COM 30980, COM 40000, COM 40200, COM 40250, COM 40350, COM 40400, COM 40450, COM 40610, and COM 50600.

The laboratory must maintain a list of modified FDAcleared assays and since this method is a modification from the manufacturer's package insert, each deviation must clearly delineated in the Standard Operating Procedure (SOP) and approved by the laboratory director. In addition, analytical validation studies are required by CAP for modified FDA-cleared tests to ensure reliable results. For qualitative interpretation, CAP requires the laboratory to verify the appropriate method specifications that are clinically relevant. In this case, quality control material and proficiency tests are used to verify the qualitative G6PD results (normal, intermediate and deficient).

Herein we report a method for immediate implementation utilizing the Abbott Architect c8000, which allows the technician to load whole blood specimens directly onto the a3600 track and subsequently review and certify the G6PD status (normal, intermediate, or deficient) and G6PD activity per gram of hemoglobin (U/g Hgb) within the laboratory information system (LIS) without having to complete any pre-analytical steps or retrieve specimens and prepare the reflex hemoglobin testing. Given a projected annual workload of 120,000 whole blood specimens in conjunction with 20 minutes of pre-analytical, specimen retrieval and reflex testing tasks, this streamlined process will eliminate 40,000 hours of manual labor annually. The concept of exploiting the on-board dilution capabilities by replacing the saline diluent on the Abbott Architect with another reagent, in this case lyse, can be implemented on other automated platforms to streamline pre-analytical steps as well.

\section{Acknowledgements}

This material has been reviewed by the Dwight $D$. Eisenhower Army Medical Center (DDEAMC). There is no objection to its presentation and/or publication. The opinions or assertions contained herein are the private views of the authors and are not to be construed as official or as reflecting true views of the Department of the Army or the Department of Defense. All experiments were conducted in a facility accredited by the College of American Pathologists. 


\section{Competing Interests}

The authors declare that they have no competing interests.

\section{Funding}

This work was supported by Defense Health Program (DHP) funds.

\section{References}

[1] Hill, D.R., Baird JK, Parise ME, Lewis LS, Ryan ET, Magill AJ, Primaquine: Report from CDC expert meeting on malaria chemoprophylaxis I. Am J Trop Med Hyg, 2006; 75(3): p. 402-415.

[2] Primaquine Phosphate Label. Food and Drug Administration Web Site http://www.accessdata.fda.gov/drugsatfda_docs/label/2015/00 8316s021lbl.pdf. Published 2015. Accessed June 1, 2018.

[3] Baird, J.K., Drug therapy: Effectiveness of antimalarial drugs. N Engl J Med, 2005; 352(15): p. 1565-1577.

[4] Updated WHO policy recommendation: Single dose primaquine as a gametocytocide in Plasmodium falciparum malaria. World Health Organization Web Site http://www.who.int/malaria/publications/atoz/who_pq_policy _recommendation/en/.

[5] Published October 2012. Accessed June 1, 2018.

[6] White, N.J., Qiao LG, Qi G, Luzzatto L., Rationale for recommending a lower dose of primaquine as a Plasmodium falciparum gametocytocide in populations where G6PD deficiency is common. Malar J, 2012; 11: p. 418.

[7] Baird, J.K., Primaquine toxicity forestalls effective therapeutic management of the endemic malarias. Int J Parasit, 2012; 42(12): p. 1049-1054.

[8] Vale, N., R. Moreira, and P. Gomes, Primaquine revisited six decades after its discovery. Eur J Med Chem, 2009; 44(3): p. 937-953.

[9] Pointe Scientific. Glucose-6-phosphate dehydrogenase reagent set. Package Insert. June 2017.

[10] Pointe Scientific. Hemoglobin Reagent. Package Insert. December 2016.

[11] Pointe Scientific. Hemoglobin Standard. Package Insert. December 2016.

[12] Trinity Biotech. Glucose-6-phosphate dehydrogenase (G-6PDH). Package Insert. February 2012.

[13] Trinity Biotech. G-6-PDH Control, Normal (G6888), Intermediate (G5029), Deficient (G5888). May 2013.

[14] BioRad. Liquichek Diabetes 172 Control Levels 1, 2 and 3. 5250B (Rev. 1). April 2014.

[15] Ambruster, D., Alexander, D., Stredler, D., Barton, C. Sample-to-Sample Carryover on an Integrated Clinical Chemistry/Immunoassay System. American Association for Clinical Chemistry Annual Meeting. June 23-27, 2006.

[16] Trinity Biotech. Architect c8000 Glucose-6-Phosphate Dehydrogenase Architect Application Catalog \# 6GLY.

[17] Pointe Scientific. Architect c8000 Glucose-6-Phosphate Dehydrogenase Application Catalog \# G7583.

[18] College of American Pathologists Accreditation Program. All Common Laboratory Checklist. August 21, 2017.

[19] College of American Pathologists Accreditation Program. Chemistry and Toxicology Checklist. August 21, 2017. 